\title{
Diseño e Implementación de un Prototipo Médico para el Tratamiento y Control de la Diabetes Mellitus Tipo 1
}

\section{Design and implementation of a medical prototype for the treatment and control of Diabetes Mellitus Type 1}

\author{
Cristian Camilo Polo Caquimbo ${ }^{[1]}$, Ricardo Quintero Rojas ${ }^{[2]}$ y Jesús David Quintero Polanco ${ }^{[3]}$
}

\begin{abstract}
Resumen
La actualización de los esquemas convencionales de tratamiento para la diabetes mellitus tipo 1 junto con el desarrollo de preparados biosintéticos más efectivos propiciaron la aparición de la terapia intensificada con insulina, que permite balancear el total de carbohidratos ingeridos por los diabéticos con la insulina administrada exógenamente, y en consecuencia, los faculta para elegir dietas más autónomas y flexibles. No obstante, la dificultad al cuantificar carbohidratos en comestibles con formas y tamaños irregulares y la consecuente inexactitud de las dosis o bolos de insulina calculados para cubrir las ingestas alimenticias, constituyen los principales inconvenientes del tratamiento desde la perspectiva de los pacientes. Este artículo muestra el diseño y construcción de un prototipo cuantificador de carbohidratos según el peso de los alimentos, pionero en la industria nacional y diseñado a la vanguardia de las nuevas tecnologías para el cuidado de la diabetes. Se presenta también una aplicación móvil asociada al dispositivo construido que proporciona una interfaz interactiva para usuarios y permite el cálculo de bolos de insulina adecuados a las necesidades del paciente. Los resultados obtenidos y los estudios científicos citados exaltan el gran potencial del prototipo desarrollado para asistir al diabético en el control de su patología.
\end{abstract}

Palabras clave: calculador de bolos; cuantificación de carbohidratos; diabetes mellitus tipo 1; terapia intensificada con insulina.

\begin{abstract}
The updating of conventional treatment schemes for diabetes mellitus type 1 together with the development of the most effective biosynthetic preparations led to the appearance of intensive insulin therapy. This type of therapy allows diabetes patients to balance their total carbohydrate intake with the insulin administered exogenously. As such, the treatment allows them to choose their diets in a way that is more autonomous and flexible. However, the difficulty of quantifying the carbohydrates in food with irregular shapes and sizes and
\end{abstract}

[1] Ingeniero Electrónico. Universidad Surcolombiana. Av. Pastrana Carrera 1a. Neiva, Huila, Colombia. E-mail: camilo.polo@hotmail.com

[2] Ingeniero Electrónico. Universidad Surcolombiana. Av. Pastrana Carrera 1a. Neiva, Huila, Colombia. E-mail: ricardo.qrl@gmail.com

[3] M. Eng. Ingeniero Electrónico. Universidad Surcolombiana. Av. Pastrana Carrera 1a. Neiva, Huila, Colombia. E-mail: jdavid@usco.edu.co

Recibido: 9 abril 2015 • Aceptado: 10 mayo 2015. 
the consequent inaccuracy of the doses of bolus insulin calculated to cover food intake, constitutes the main inconvenience of the treatment from the patients' perspective. This article presents the design and construction of a prototype carbohydrate counter that quantifies the carbohydrates according to the weight of the food. Such a design is new in the national industry and is at the forefront of new technology for diabetes. We also present a mobile application associated to the device that provides an interactive interface for users allowing them to calculate the insulin bolus according to their needs. The results obtained and the scientific studies cited exalt the great potential of this prototype to help diabetes patients control their pathology.

Key words: bolus calculator, carbohydrate counting, diabetes mellitus type 1, intensive insulin therapy.

\section{Introducción}

La diabetes mellitus tipo 1 es una enfermedad de etiología desconocida causada por la destrucción de las células productoras de insulina por parte del sistema inmunitario (Alba et al, 2004), se caracteriza por un déficit significativo de esa hormona en el paciente diabético e implica la dependencia vitalicia de insulina exógena (Olvera Granados et al, 2008). Su tratamiento se basa en el equilibrio de la alimentación, los fármacos y el ejercicio físico regular junto con una adecuada educación diabetológica, de manera que tanto el paciente como su familia dispongan de las habilidades y conocimientos necesarios para gestionar el día a día de la diabetes (Jansà et al, 2011).

En concordancia con los resultados del Diabetes Control and Complications Trial (DCCT) así como de otros estudios posteriores, la terapéutica más adecuada para esa patología se ciñe al esquema "basal - bolo", que trata de reproducir el patrón de secreción endógena de insulina de una forma más precisa que la terapia convencional (Sáez de la Fuente et al, 2008) y provee, entre muchas otras ventajas, unos valores medios más bajos de hemoglobina glicosilada (HbA1c), lo cual se asocia a una reducción de las complicaciones microvasculares (The Diabetes Control and Complications Trial Research Group, 1993; Bode et al, 1996; Rudolph e Hirsch, 2002); y procura para el diabético un estilo de vida más flexible en relación con su dieta, horarios y actividad física (Evans et al, 1999; Karter et al, 2001).

No obstante, las mayores dificultades a la hora de alcanzar un control óptimo con este tipo de terapia están relacionadas con el aumento del riesgo de hipoglucemia (debido a que el control intensivo de la glucosa en sangre tiende a alcanzar niveles más bajos (Davis y Alonso, 2004)) y con un mayor esfuerzo por parte del paciente al cuantificar carbohidratos en sus alimentos y al incrementar el número de administraciones de insulina y de controles diarios de glucemia capilar, que requieren una educación diabetológica más exhaustiva (Skyler, 1997).

En vista de ese panorama y al considerar que en el mercado nacional no existe un dispositivo médico que integre la cuantificación de carbohidratos en alimentos con el calculador de bolos, se diseñó e implementó un prototipo no invasivo que determina el total de carbohidratos de un alimento o comida, y calcula, mediante la interacción con una aplicación móvil asociada, la cantidad de insulina adecuada para cubrir su ingesta y para corregir el nivel de glucosa sanguíneo del paciente si fuere necesario.

\section{Escenario de aplicación}

Los altos costo económicos de la diabetes y sus complicaciones vistos desde la perspectiva de los sistemas públicos de salud han propiciado el desarrollo de mecanismos de tratamiento más rentables y eficaces para el control de esa patología.

En la actualidad, la estrategia terapéutica que más se adapta a ese modelo es la insulinoterapia intensificada con conteo de hidratos de carbono, pues entre las ventajas del conteo de hidratos de carbono se destaca que permite mayor flexibilidad en la elección de los alimentos, mejora el control de las glucemias, y el plan alimentario se adapta mejor a los horarios cambiantes de trabajo u otras actividades, lo que aporta una mejor calidad de vida (Laurenzi et al, 2011); pese a que su utilización puede resultar dificultosa cuando los alimentos consumidos varían en tamaño, como las frutas y la hogaza del pan (UCSF Medical Center, 2008), o en aquellos alimentos que no pueden medirse fácilmente como cereales para el desayuno, arroz cocido o pasta (Fernández et al, 2013).

Asimismo, los beneficios que proveen los ajustes prandiales de insulina, que se realizan en función de la 
glicemia preprandial y del contenido de carbohidratos de la ingesta alimenticia (DeWitt \& Hirsch, 2003), pueden resultar infructíferos cuando se desencadenan hipoglicemias en los diabéticos por causa de errores en cálculos matemáticos o falencias en la fundamentación teórica sobre el tratamiento para la patología, al punto que, según un estudio publicado en 2010, un 64\% de las personas con diabetes tipo 1 calculan mal los bolos de insulina que deben administrarse diariamente en las comidas, con consecuencias negativas a corto y largo plazo para su salud (Moreiro Socías, 2012).

En tal virtud, se diseñó e implementó un prototipo médico dirigido a pacientes diabéticos tipo 1 que utilicen la terapia intensificada con insulina como esquema de control de su enfermedad y deseen optimizar el proceso de conteo de carbohidratos en comestibles que no poseen etiquetas de información nutricional.

Al considerar que los hábitos alimenticios de la población insulinorequiriente del país están claramente definidos, se determinó la elección de 135 alimentos no procesados industrialmente y seleccionados de entre los principales comestibles de consumo nacional para proveer la base de datos del dispositivo cuantificador de carbohidratos. La información nutricional de cada alimento se obtuvo de la Tabla de Composición de Alimentos de América Latina publicada por la Oficina Regional de la FAO para América Latina y el Caribe y del Sistema de Alimentos Equivalentes publicado por la Escuela de Nutrición y Dietética de la Universidad Industrial del Santander.

\section{Materiales y métodos}

El prototipo diseñado (en lo sucesivo denominado “CHO QUANTIFIER") permite mediante la interacción de un sistema electrónico de pesaje y una aplicación homónima ejecutable en cualquier dispositivo móvil con sistema operativo Android (Figura 1), determinar la cantidad exacta de carbohidratos contenidos en un alimento y la dosis de insulina adecuada para cubrir su ingesta de conformidad con los lineamientos de la terapia intensificada con insulina.

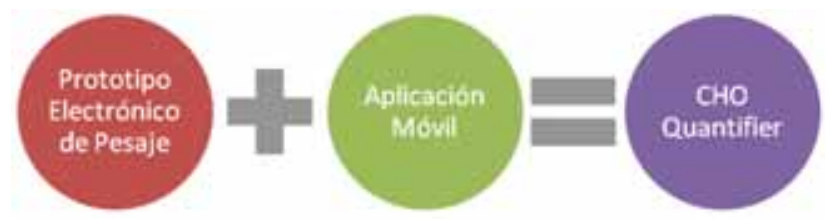

Figura 1. Esquema operativo del dispositivo "CHO QUANTIFIER”.

\subsection{Prototipo electrónico de pesaje}

Es el componente físico del dispositivo "CHO QUANTIFIER" que permite cuantificar la masa (medida de la cantidad de materia) de los alimentos objeto de estudio. Su modelo de operación se basa principalmente en la interacción de una celda de carga y un puente de Wheatstone (Espinosa Esquivel, 1995), pues permiten obtener un nivel de tensión proporcional a la fuerza ejercida por los objetos analizados.

La celda de carga elegida está construida en aluminio, es idónea para trabajo en ambientes secos y posee una capacidad máxima $\left(E_{\max }\right)$ de 5 kg y unos límites de sobrecarga y ruptura que posibilitan la integración de variados menús alimenticios sin poner en riesgo el funcionamiento del prototipo construido por exceder los límites de peso soportados.

Con el propósito de incrementar la tensión de salida de la celda de carga hasta un nivel apropiado para satisfacer los requerimientos de la conversión analógica digital, se empleó el circuito integrado HX711, que corresponde a un ADC de 24 bits especialmente diseñado para escalas de pesaje (Figura 2) y aplicaciones de control industrial que interactúan directamente con puentes de medición.

El integrado posee dos canales de entrada diferencial con bajo ruido y amplificación de ganancia programable de hasta 128 cuando se utiliza el Canal A con una alimentación de $5 \mathrm{~V}$ en el pin AVDD (pin de alimentación analógica), que posibilita la amplificación de la señal proveniente de la etapa de sensado hasta un nivel de $1,15 \mathrm{~V}$.

La resolución del dispositivo, entretanto, se obtiene en términos del voltaje de referencia y del número de bits del conversor (Lockhart, 2014) como sigue a continuación:

$$
\text { Resolución }=\frac{V_{D}}{V_{S} \cdot 2^{(n-B)}} \cdot E
$$

Donde,

$V_{D}$ :Rango a escala completa del voltaje de entrada del conversor.

$V_{S}$ : Rango de tensión de salida a escala completa de la señal a medir.

E: Escala completa de la variable a medir.

$n$ : Número de bits del conversor.

$B$ : Gama del conversor, unipolar $(B=0$, puede medir señales que están por encima o por debajo de cero, pero no al mismo tiempo) o bipolar ( $\mathrm{B}=1$, puede medir señales por encima y por debajo de cero). 


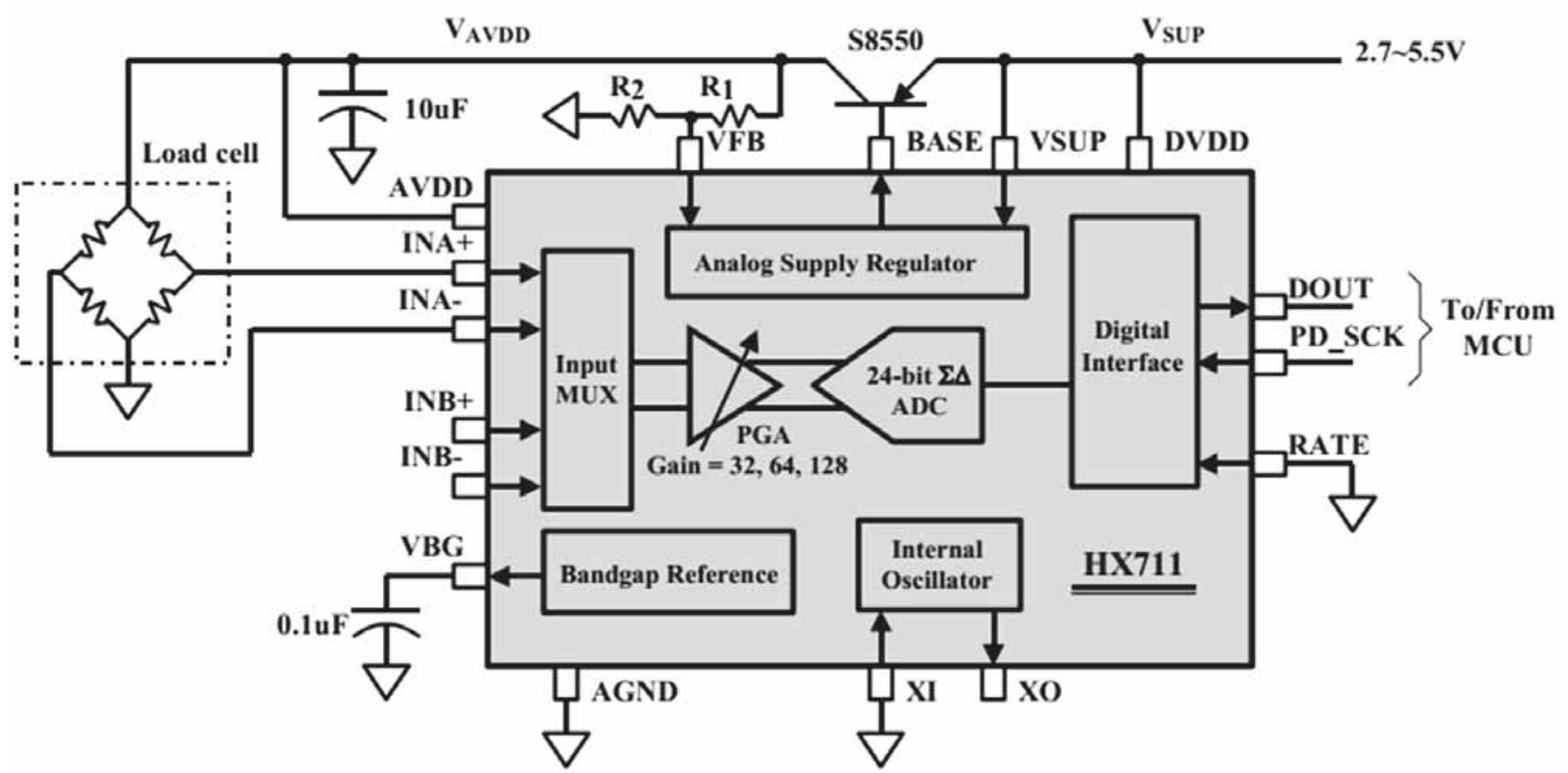

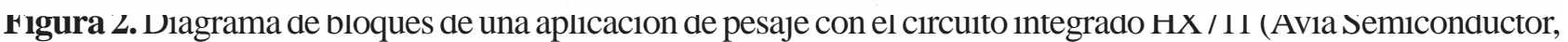
2012).

No obstante y con el propósito de evitar imprecisiones debidas al uso injustificado de bits en el conversor, se determinó mediante software el truncamiento de los 6 bits menos significativos del dispositivo; obteniéndose, con un valor de $V_{D}$ igual a $5 \mathrm{~V}$ y de $V_{S}$ igual a $1,15 \mathrm{~V}$ que:

$$
\text { Resolución }=\frac{5 \mathrm{~V}}{1,15 \mathrm{~V} \cdot 2^{((24-6)-1)}} \cdot 5000 \mathrm{~g}=0,17 \mathrm{~g}
$$

Valor que resulta muy apropiado para los requerimientos del prototipo, pues el peso de un confite en presentación individual comúnmente sobrepasa los 5 g, es decir, aproximadamente 30 veces la resolución del dispositivo; más aún, resulta muy viable al considerar que un menú variado concentra aproximadamente $450 \mathrm{~g}$ de alimentos por persona (Nutrition Advice Service, 2013).

Para interpretar y ejecutar las instrucciones que permiten la toma de decisiones lógicas relacionadas con el funcionamiento del "CHO QUANTIFIER" se recurrió al módulo Teensy 3.0, que se basa en un microcontrolador ARM Cortex M4 de 32 bits y posee características como bajo consumo energético, tamaño reducido y diseño que permite aprovechar la gran capacidad de computación y características periféricas y lo convierten en un dispositivo muy atractivo frente a otras placas de desarrollo (Baraka et al, 2013).

Adicionalmente y con el propósito de permitir la interacción entre los usuarios y el "CHO QUANTIFIER” se incluyó en el prototipo electrónico de pesaje un panel táctil que despliega los controles físicos de Auto Cero (ZERO), Adicionar Alimentos (ADD) y Calibración (CAL), implementados a través de la utilización de tres de los nueve pines táctiles que provee la placa Teensy 3.0, cuyo funcionamiento se centra en la utilización de la librería 'CapacitiveSensor' que convierte algunos pines específicos del módulo Teensy 3.0 (Figura 3) en sensores capacitivos, los cuales pueden detectar la capacitancia eléctrica del cuerpo humano.

En vista de la difusión y masivo consumo de dispositivos recargables, se determinó la implementación de un módulo de abastecimiento eléctrico basado en una batería Ni-MH (Níquel Metal Hidruro) recargable de 9 V y 250 mA, que ofrece mayor capacidad, mayor tiempo de vida y soporta más recargas si se le compara con una batería tradicional de NiCd, y convierte al “CHO QUANTIFIER" en un equipo portátil que colabora con la conservación medioambiental (ya que no utilizan materiales pesados, como el cadmio (Informática Hoy, 2008)) y permite al usuario final obtener un importante ahorro de dinero por su utilización. Se incluyó también en el diseño de la etapa un cargador de intensidad constante adecuado para efectuar recargas eficientes y dirigido a procurar la máxima duración de las baterías.

Por otro lado y en vista de las diversas maneras que actualmente existen para conectar dispositivos electrónicos entre sí y la variedad incluso mayor de protocolos disponibles que fomentan la implementación de modelos 


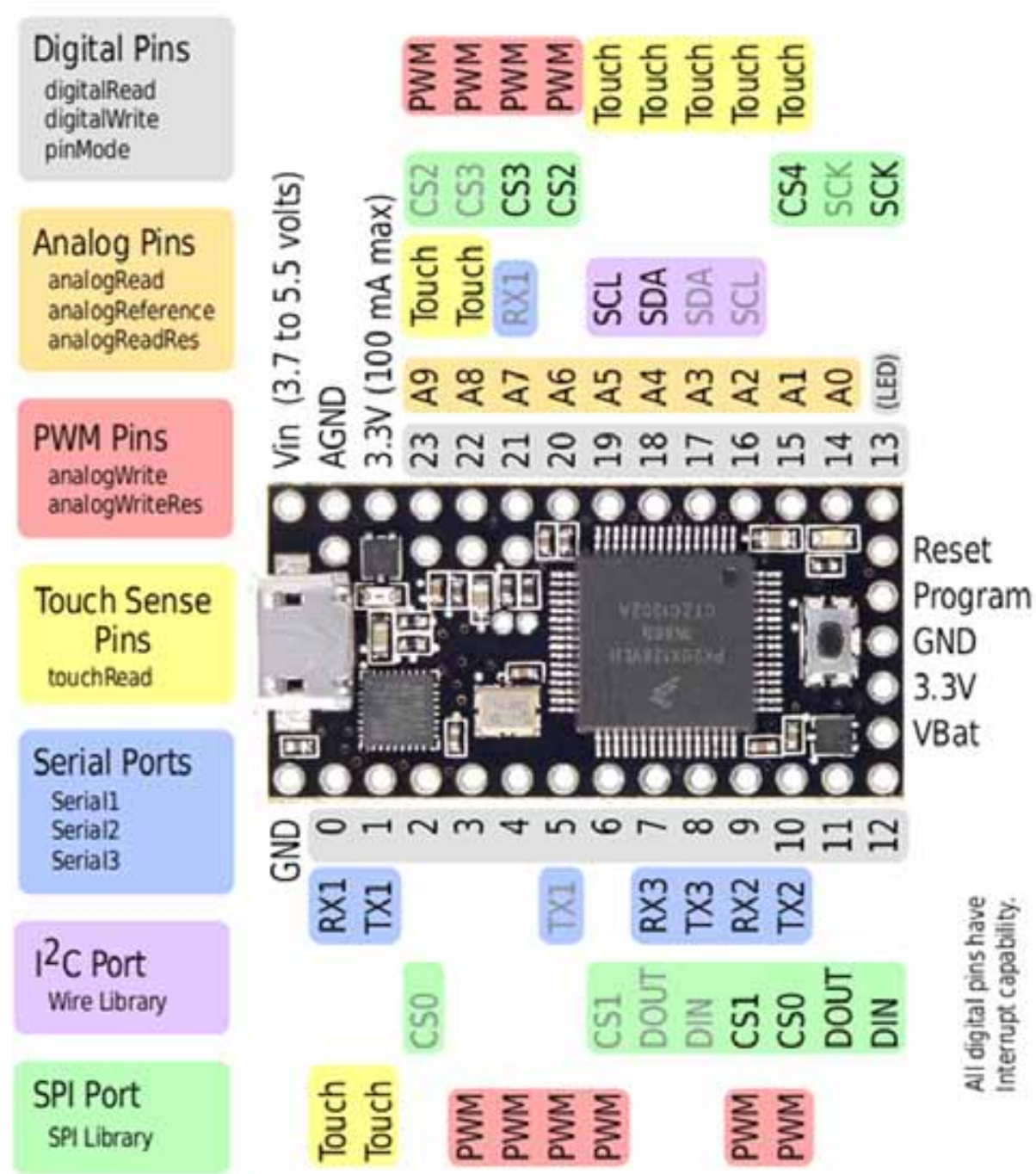

Figura 3. Diagrama de conexión del módulo Teensy 3.0 (Stoffregen, 2012).

de comunicación inalámbricos, los cuales son cada vez más utilizados porque impulsan la movilidad y la ubicuidad en todo tipo de comunicación, se determinó la utilización del módulo Bluetooth BlueSMiRF Silver.

Una de las principales ventajas del módulo, además de su pequeño tamaño $(0,38 \mathrm{~cm}$ x $1,52 \mathrm{~cm}$ x $4.83 \mathrm{~cm})$ y sus buenas características de transmisión y recepción que le brindan un alcance muy amplio, es el bajo consumo de corriente que posee tanto en funcionamiento, como en modo de espera, es decir, alimentado con energía pero sin conexión o enlace a otro dispositivo. Otro detalle particular es que su bajo voltaje de alimentación permite que sea abastecido eléctricamente durante muchas horas por baterías recargables o alcalinas, demostrando características excepcionales en aplicaciones médicas o para actividades donde la fuente energética debe ser liviana y portátil (Spark Fun Electronics, 2013).

\subsection{Aplicación móvil}

Una aplicación móvil es un programa que se puede descargar y al que se puede acceder directamente desde un dispositivo inteligente o desde algún otro aparato móvil (Comisión Federal de Comercio del Gobierno de los Estados Unidos, 2011), su utilidad es mucho mayor que la de una web convencional y, a fin de cuentas, es una herramienta más que pretende simplificar las tareas de sus usuarios (Bonías, 2013).

Dado tal escenario y considerando que Android es el Sistema Operativo más utilizado en la actualidad, el diseño y desarrollo de la aplicación móvil adscrita al “CHO QUANTIFIER” se fundamentó en ese Sistema Operativo, para lo cual se empleó el IDE (Entorno de Desarrollo Integrado) Android Studio en la versión 0.4.6., que ofrece nuevas y potentes herramientas para el desarrollo de aplicaciones móviles. 
Simultáneamente y con el propósito de estructurar la información nutricional necesaria para el funcionamiento del "CHO QUANTIFIER" y de suministrarla correctamente a la aplicación móvil, se empleó el Lenguaje de Consulta Estructurado o SQL (por sus siglas en inglés Structured Query Language) que permite el acceso a bases de datos relacionales, así como efectuar consultas con el fin de recuperar información y hacer cambios de forma sencilla.

\section{Funcionamiento}

Los modos de operación provistos por la aplicación móvil asociada al "CHO QUANTIFIER” permiten la diferenciación de usuarios como se detalla continuación:

\subsection{Usuario diabético tipo 1}

Se rige por los preceptos médicos de la terapia intensiva con insulina y permite el registro de usuarios siempre que se especifiquen los datos personales del paciente y los parámetros de Sensibilidad (que indica cuánta glucosa es metabolizada por unidad de insulina) y Ratio (que indica cuántos carbohidratos cubre una unidad de insulina), a fin de determinar la dosis de medicación adecuada para cubrir los carbohidratos contenidos en la comida analizada y/o para corregir el nivel de glucosa sanguíneo (GS) del paciente, siempre que él especifique su nivel actual de glucosa en sangre (Figura 4), conforme a la siguiente ecuación:
Dosis Insulina $=$

Bolo alimenticio + Bolo corrección

Dosis Insulina $=$

$$
\frac{\text { CHO alimentos }}{\text { Ratio }}+\frac{\text { GS actual }- \text { GS deseada }}{\text { Sensibilidad }}
$$

En este modo de operación es posible agregar alimentos al recuento total mediante la función ADD y registrar los carbohidratos de comestibles que poseen etiquetas de información nutricional o que no están incluidos en la base de datos del dispositivo.

Adicionalmente, provee un historial que presenta información estadística por usuario sobre carbohidratos ingeridos, bolos de insulina y registros de glucosa sanguínea.

\subsection{Usuario no diabético}

Se focaliza en familiares o allegados de pacientes diabéticos insulinorequirientes que desean hacer hincapié en el conteo de carbohidratos. Permite, al igual que en el modo anterior, el registro de usuarios cuando éstos proveen su información personal.

Para calcular el contenido de carbohidratos de una comida en este modo de operación se emplea la misma metodología del modo "Usuario diabético tipo 1", sin embargo, aquí no se calculan las dosis de insulina

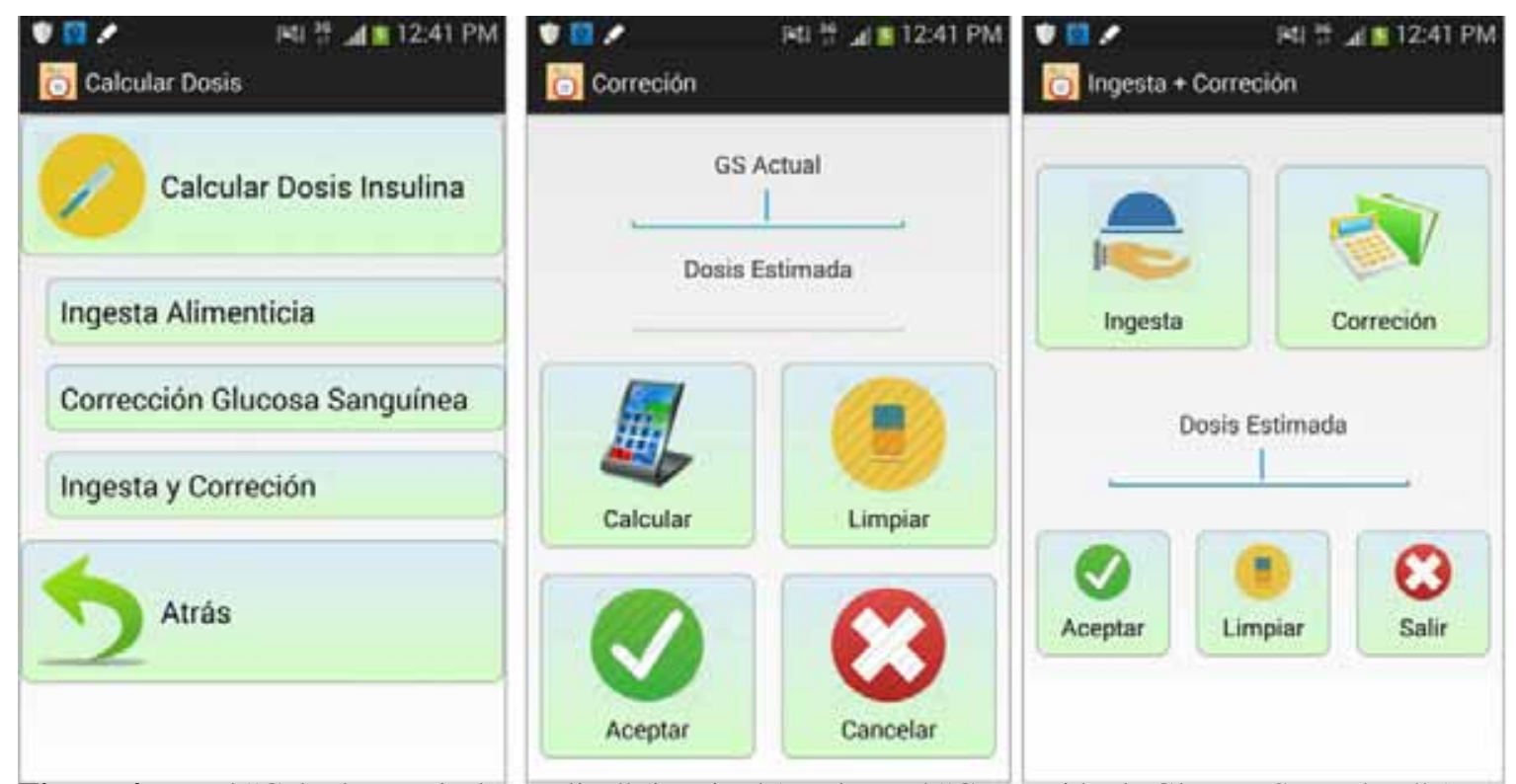

Figura 4. Menú “Calcular Dosis de Insulina” (izquierda), submenú “Corrección de Glucosa Sanguínea” (centro) y submenú “Ingesta y Corrección ” (derecha). 
para cubrir la ingesta de los alimentos analizados ni para corregir el nivel de glucosa sanguíneo, pues para efectuar tales operaciones es indispensable conocer los valores de Sensibilidad y Ratio, siendo claro que son parámetros exclusivos del diabético y varían de un paciente a otro.

\section{Resultados}

Tomando como referente el régimen alimenticio de un diabético tipo 1 , se efectuó una comparación entre el total de carbohidratos calculado por el paciente a través de las listas de intercambios de carbohidratos y el total de carbohidratos cuantificado por el dispositivo "CHO QUANTIFIER” en cada una de las seis comidas del día.
El análisis de los datos obtenidos arrojó un rango de diferencias entre mediciones de (0.3-6.1) g y una diferencia máxima porcentual entre mediciones de $16.9 \%$, que revela significativas falencias en los actuales métodos de cuantificación de carbohidratos y resalta la funcionalidad del prototipo construido.

Obteniéndose, de tal manera, un prototipo médico no invasivo basado en los lineamientos más vanguardistas para el cuidado de la diabetes y pionero en la industria nacional al integrar estratégicamente en un mismo dispositivo el cuantificador de carbohidratos en alimentos junto con el calculador de bolos (Figura 5), que hasta hace poco sólo estaba disponible con los sistemas de infusión continuos de insulina (Moreiro Socías, 2012).

\begin{tabular}{|c|c|c|c|c|c|c|}
\hline & Desayuno & Nueves & Almuerzo & Onces & Cena & Trasnocho \\
\hline 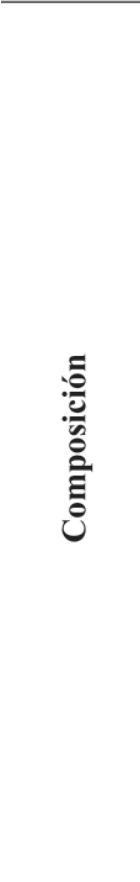 & $\begin{array}{l}2 \text { rebanas de pan } \\
\text { integral. } \\
1 \text { lonja de jamón. } \\
1 \text { lonja de queso } \\
\text { entero. } \\
2 \text { rodajas de } \\
\text { tomate. } \\
1 \text { pocillo de café } \\
\text { con leche. }\end{array}$ & $\begin{array}{l}1 / 2 \text { porción } \\
\text { de mago. } \\
1 / 2 \text { porción } \\
\text { de manzana } \\
\text { verde. }\end{array}$ & $\begin{array}{l}2 \text { unidades de } \\
\text { papa sabanera } \\
\text { cocida. } \\
\text { 1/2 taza de } \\
\text { arroz blanco. } \\
1 \text { porción de } \\
\text { carne magra } \\
\text { asada. } \\
1 \text { plato postre } \\
\text { de ensalada } \\
\text { verde (lechuga } \\
\text { batavia, } \\
\text { tomate verde, } \\
\text { maíz tierno y } \\
\text { cebolla } \\
\text { cabezona). } \\
1 \text { vaso de jugo } \\
\text { (elaborado con } \\
\text { una porción de } \\
\text { piña). }\end{array}$ & $\begin{array}{l}1 \text { vaso de } \\
\text { yogurt sin } \\
\text { azúcar - } \\
\text { producto con } \\
\text { etiqueta de } \\
\text { información } \\
\text { nutricional }\end{array}$ & $\begin{array}{l}1 \text { papa cocida } \\
\text { en cuadros. } \\
1 / 2 \text { taza de } \\
\text { pollo } \\
\text { desmechado. } \\
1 / 4 \text { de taza de } \\
\text { habichuela. } \\
1 / 4 \text { de } \\
\text { zanahoria. } \\
1 \text { vaso de jugo } \\
\text { (elaborado con } \\
\text { dos } \\
\text { mandarinas } \\
\text { promedio). }\end{array}$ & $\begin{array}{l}1 \text { vaso de leche } \\
\text { descremada. } \\
2 \text { galletas de soda. }\end{array}$ \\
\hline 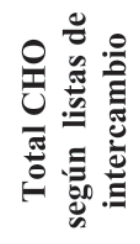 & $40 \mathrm{~g}$ & $30 \mathrm{~g}$ & $72.5 \mathrm{~g}$ & $12 \mathrm{~g}$ (etiqueta) & $36 \mathrm{~g}$ & $20 \mathrm{~g}$ \\
\hline 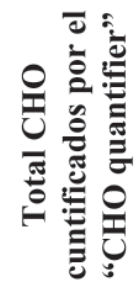 & $42.6 \mathrm{~g}$ & $36.1 \mathrm{~g}$ & $67.9 \mathrm{~g}$ & $12 \mathrm{~g}$ (etiqueta) & $42.4 \mathrm{~g}$ & $19.7 \mathrm{~g}$ \\
\hline
\end{tabular}




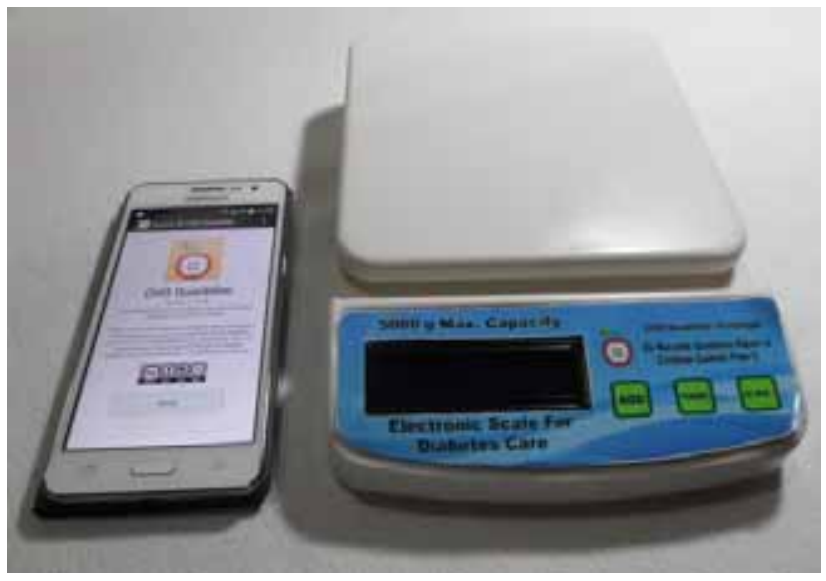

Figura 5. Dispositivo “CHO QUANTIFIER”.

La aplicación móvil desarrollada (ver créditos de autoría en la Figura 6) provee una interfaz interactiva para usuarios a través de dos modos de operación bien diferenciados que pretenden satisfacer las necesidades individuales de cada usuario y permitir la bidireccionalidad en la cuantificación de carbohidratos de una comida.

Así pues, al considerar que la inexactitud en el conteo de carbohidratos representa la principal dificultad del paciente diabético al calcular sus dosis de insulina, cobra validez la tasación que exalta al " $\mathrm{CHO}$ QUANTIFIER” como “un punto importante e innovador en la tarea de lograr simplificar y optimizar el tratamiento del paciente diabético mejorando su calidad de vida” (Quero Acosta, 2014).

En tal virtud, la correcta utilización del dispositivo “CHO QUANTIFIER” podría facilitar el cálculo de los bolos de insulina en comparación con el cálculo manual, además de mejorar la confianza del paciente en la exactitud de las dosis de medicación que se debe administrar y reducir el temor a los episodios de hipoglucemia en un $89,3 \%$, en concordancia con un reciente estudio realizado con más de 1.400 pacientes diabéticos del Reino Unido e Irlanda que arrojó estos resultados y recogió la utilidad y beneficios del uso del calculador de bolos (Moreiro Socías, 2012).

Por otro lado, el conteo de hidratos de carbono puede incrementar el buen control de la glucemia, lo que resulta en una disminución de la hemoglobina glicosilada (HbA1c) y así menos complicaciones, pues se comprobó que reduciendo el $\mathrm{HbA} 1 \mathrm{c}$ en un 1\% en pacientes diabéticos tipo 1 se pueden reducir las complicaciones de retinopatías en un 38\%, de neuropatías en un 28\% y de nefropatías en un 35\% (Freed y Joffe, 2000).
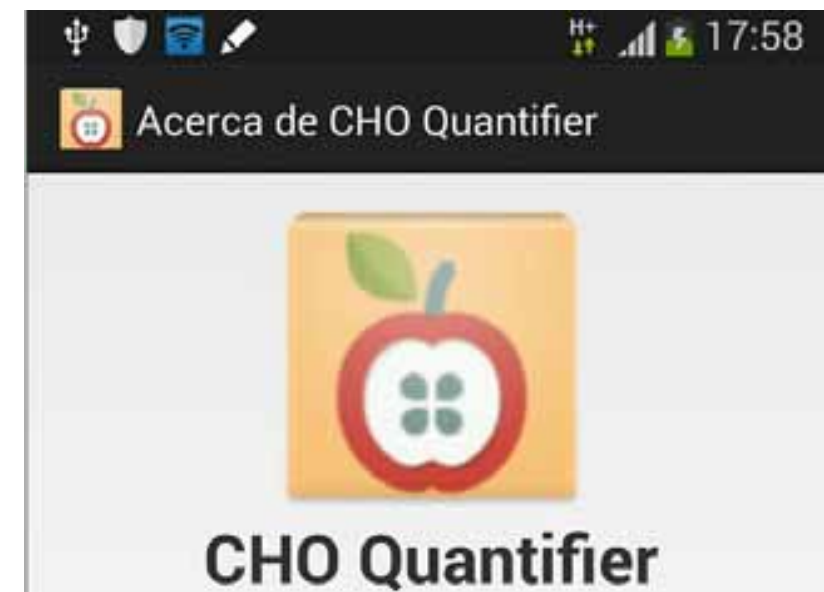

\section{CHO Quantifier}

\author{
Versión 1.12.36 \\ Desarrollado por Camilo Polo y Ricardo Quintero \\ Creative Commons License
}

Usted es libre de copiar y compartir esta aplicación móvil siempre y cuando proporcione los creditos de autoría correspondientes, la utilice para fines no comerciales y disponga de ella en su estado original, sin transformación o modificación alguna.

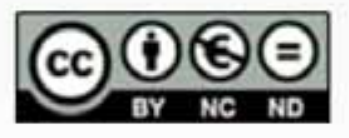

\section{Atrás}

Figura 6. Pantalla de créditos de la aplicación " $\mathrm{CHO}$ QUANTIFIER”.

\section{Discusión}

La diabetes se perfila en la actualidad como uno de los grandes retos para la salud pública, tanto en países desarrollados como en países de ingresos medios y bajos. De acuerdo con la Organización Mundial de la Salud (OMS), la diabetes afecta entre un 10\% y 15\% de la población adulta de América Latina y el Caribe y se estima que para el 2025 la prevalencia alcance los 65 millones de personas (Dávila Cervantes et al, 2011).

En el caso de Colombia, la prevalencia estimada de la DM1 para el 2009 correspondía al 0.07\%, equivalente a un total nacional cercano a los 30.000 individuos (Vargas Uricoechea, 2011); y según datos de 2007 reportados por la Federación Internacional de Diabetes, la prevalencia nacional de la DM2 correspondía al 4.8\% de la población, equivalente a 1.427.300 personas diagnosticadas (Peña Torres, 2011). 
Dentro de este contexto, resulta también importante conocer la carga monetaria que esta enfermedad representa, pues en el año 2000, se estimó el costo atribuido a la DM en 2.586,8 millones de dólares, de los cuales 2.172 millones correspondieron a costos indirectos (por mortalidad prematura y discapacidad debidas a la DM) y 415 a costos directos (por medicación, hospitalizaciones, consultas y complicaciones derivadas de la DM) (Tamayo, 2013).

En vista de tal panorama, se pretende con la utilización del prototipo “CHO QUANTIFIER” impactar a las personas nacionales diagnosticadas con DM1 que utilizan el tratamiento intensificado con insulina y a las diagnosticadas con DM2 que emplean el tratamiento suplementario con insulina para el control de su patología.

Asimismo, se plantean los cimientos para la realización de un estudio clínico que permita validar y cuantificar los beneficios aportados por el " $\mathrm{CHO}$ QUANTIFIER” en la optimización de la terapéutica de la DM1.

\section{Conclusiones}

El prototipo construido pone al alcance del paciente diabético una novedosa herramienta para determinar de forma muy fácil e intuitiva la cantidad exacta de carbohidratos contenidos en sus alimentos (siempre que su información nutricional se encuentre precargada en la base de datos del dispositivo) y la dosis de insulina adecuada para cubrir su ingesta o para corregir elevaciones en su nivel de glucosa sanguíneo si fuere necesario.

El dispositivo implementado es asequible a la comunidad interesada, responde adecuadamente a los requerimientos de la población diabética insulinorequiriente y propicia en los pacientes que utilizan la terapia intensiva con insulina un mayor control de sus niveles de glucosa sanguínea.

La utilización del “CHO QUANTIFIER” dentro de un tratamiento asistido por profesionales médicos puede propiciar una significativa mejoría en el control de glucosa sanguínea de los diabéticos y al considerar la carga monetaria que la diabetes representa desde la perspectiva del sistema de salud y desde la perspectiva del propio paciente, implicaría una sustancial reducción en los recursos económicos destinados a las complicaciones derivadas de esta enfermedad.
Con base en los estudios científicos que revelan la utilidad del conteo de carbohidratos y del calculador de bolos en el tratamiento de la diabetes mellitus tipo 1 , es posible afirmar que el "CHO QUANTIFIER" es una herramienta con gran potencial para asistir a los pacientes diabéticos en el control de su patología.

\section{Nota aclaratoria}

Los planteamientos y resultados obtenidos a partir del desarrollo del dispositivo presentado pertenecen exclusivamente a los estudiantes Cristian Camilo Polo Caquimbo y Ricardo Quintero Rojas, realizadores del presente trabajo de investigación, y en tal virtud, los derechos morales y patrimoniales que la ley otorga. Cualquier modificación o actualización realizada a este trabajo de investigación deberá respetar los correspondientes derechos de autoría.

\section{Referencias bibliográficas}

1. Alba, A., Verdaguer, J., Vives, M., 2004. Diabetes Mellitus Tipo 1: Autoinmunidad frente a la Célula Beta. Endocrinología y Nutrición. 51 (3), 121-125.

2. Avia Semiconductor, 2012. HX711: 24-Bit Analogto-Digital Converter (ADC) for Weigh Scales. Consultado el 6 de mayo de 2014. http://www.dfrobot. com/image/data/SEN0160/hx711_english.pdf

3. Baraka, K., Ghobril, M., Malek, S., Kanj, R., Kayssi, A., 2013. Low Cost Arduino/Android-Based EnergyEfficient Home Automation System with Smart Task Scheduling. Fifth International Conference on Computational Intelligence, Communication Systems and Networks (CICSyN). IEEE. 296-301.

4. Bode, B. W., Steed, R. D., Davidson, P. C., 1996. Reduction in Severe Hypoglycemia with Long-term Continuous Subcutaneous Insulin Infusion in Type 1 Diabetes. Diabetes Care. 19, 324-327.

5. Bonías, C., 2013.¿Cuáles son las Ventajas de Tener una Aplicación Móvil?. Nubelo Solutions.

6. Comisión Federal de Comercio, 2011. Cómo Funcionan las Aplicaciones Móviles: Preguntas y Respuestas. AlertaenLinea.gov (sitio web del Gobierno Federal de los Estados Unidos).

7. Dávila Cervantes, C. A., Agudelo Botero, M., Gloria Hernández, L. E., 2011. Diabetes en México y 
Colombia: Análisis de la Tendencia de Años de Vida Perdidos, 1998-2007. Revista de Salud Pública. México D.F. 43, 560-571.

8. Davis, S., Alonso, M. D., 2004. Hypoglycemia as a Barrier to Glycemic Control. Journal of Diabetes and its Complications. 18, 60-68.

9. DeWitt, D. E., Hirsch, I. B., 2003. Outpatient Insulin Therapy in Type 1 and Type 2 Diabetes Mellitus: Scientific Review. The Journal of the American Medical Association. 289, 2254-2264.

10.Espinosa Esquivel, M., 1995. Diseño y Construcción de una Celda de Carga. Tesis de Grado, Universidad Autónoma de Nuevo León - División de Estudios de Post-grado.

11.Evans, J. M., Newton, R. W., Ruta, D. A., MacDonald, T. M., Stevenson, R. J., Morris, A. D., 1999. Frequency of Blood Glucose Monitoring in Relation to Glycaemic Control: Observational Study with Diabetes Database. British Medical Journal. 319, 83-86.

12.Fernández, N., Sverdlick, A., Rosón, M. I., Presner, N., González Infantino, C., 2013. Tratamiento con Múltiples Dosis de Insulina y Conteo de Hidratos de Carbono. Su Relación con el Aumento de Peso, Frecuencia de Hipoglucemia y Control Glucémico en una Población de Adultos con Diabetes Tipo 1. Actualización en Nutrición. 14 (3), 201-210.

13.Freed, S. H., Joffe. D. J., 2000. The Clinical Impact of Fiber Supplementation for the Reduction of Postprandial Blood Glucose and Risk Reduction of Complications from Diabetes. Diabetes in Control. 1, 12-18.

14.Informática Hoy, 2008. Electrónica de Consumo Masivo: Pilas Recargables. Argentina.

15.Jansà, M., Murillo, S., Vidal, M., 2011. Medical Nutritional Therapy and Therapeutic Patient Education in Diabetes. Revista de Enfermería. 34 (5), 16-23.

16.Karter, A. J., Ackerson, L. M., Darbinian, J. A., D’Agostino Jr, R. B., Ferrara, A., Liu, J., 2001. Selfmonitoring of Blood Glucose Levels and Glycemic Control: the Northern California Kaiser Permanente Diabetes Registry. The American Journal of Medicine. 111, 1-9.
17.Laurenzi, A., Bolla, A. M., Panigoni, G., Doria, V., Uccellatore, A., Peretti, E., Saibene, A., Galimberti, G., Bosi, E., Scavini, M., 2011. Effects of Carbohydrate Counting on Glucose Control and Quality of Life Over 24 Weeks in Adult Patients With Type 1 Diabetes on Continuous Subcutaneous Insulin Infusion: a Randomized, Prospective Clinical Trial (GIOCAR). Diabetes Care. 34 (4), 823-827.

18.Lockhart, R. W., 2014. How Much ADC Resolution do You Really Need?. DataQ Instruments, Inc.

19.Moreiro Socías, J., 2012. El Calculador de Bolo: Optimización de la Terapia en la Diabetes Tipo 1. Diabéticos Presente \& Futuro. Accu-Chek Roche. Ed. Mayo, S.A. Mallorca. 81, 4-6.

20.Nutrition Advice Service, 2013. Portion Sizes: Servings and Portions. Waitrose. London, UK.

21. Olvera Granados, C. P., Leo Amador, G. E., Hernández Montiel, H. L., 2008. Páncreas y Células Beta: Mecanismos de Diferenciación, Morfogénesis y Especificación Celular Endocrina. ¿¿Regeneración?. Boletín Médico del Hospital Infantil de México. 65 (4), 306-324.

22.Peña Torres, E., 2011. Celebración del día Internacional de la Diabetes. Ministerio de Salud y la Protección Social de la República de Colombia.

23. Quero Acosta, L., 2014. Talking about the Carbohydrates Counting. Clínica Santa Isabel ASISA. Sevilla, España.

24. Rudolph, J. W., Hirsch, I. B., 2002. Assessment of Therapy with Continuous Subcutaneous Insulin Infusion in an Academic Diabetes Clinic. Endocrine Practice. 8, 401-405.

25.Sáez de la Fuente, J., Granja Berna, V., Ferrari Piquero, J. M., Valero Zanuy, M. A., Herreros de Tejada López-Coterilla, A., 2008. Tipos de Insulinoterapia. Revista Clínica Española. 208 (2), 76-86.

26.Skyler, J. S., 1997. Tactics for Type 1 Diabetes. Endocrinology Metabolism Clinics of North America. 26 (3), 647-656. 